\title{
A Network Pharmacology Approach to Understanding the Mechanisms of Action of Traditional Medicine: Rheum L. for Diabetic Kidney Disease
}

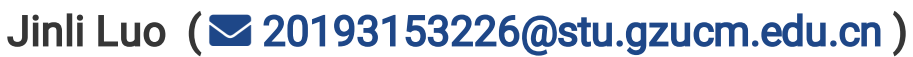

Guangzhou University of Chinese Medicine

\section{Chunli Piao}

Shenzhen Hospital『 Guangzhou University of Chinese Medicine『Futian》

De Jin

China Academy of Chinese Medical Sciences Guanganmen Hospital

\section{Li Wang}

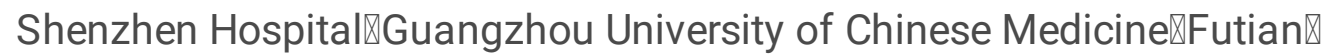

\section{Xiaohua Zhao}

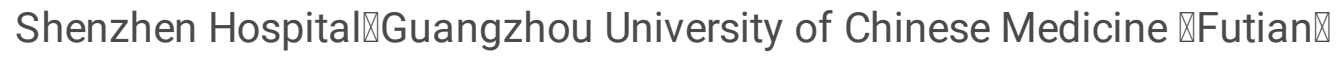

\section{Fengmei Lian}

China Academy of Chinese Medical Sciences Guanganmen Hospital

\section{Xiaolin Tong}

China Academy of Chinese Medical Sciences Guanganmen Hospital

\section{Research}

Keywords: Rheum L., Network pharmacology, Diabetic kidney disease

Posted Date: October 14th, 2020

DOI: https://doi.org/10.21203/rs.3.rs-76167/v1

License: (a) (i) This work is licensed under a Creative Commons Attribution 4.0 International License. Read Full License 


\section{Abstract}

\section{Background}

Rheum L. (Da-huang in pinyin, Radix Rhei Et Rhizome in pharmaceutical name), a classic Chinese herb, has been extensively used to treat diabetic kidney disease in clinical practice in China for many years. However, the pharmacological mechanisms of Rheum L. remain elusive. To decrypt the underlying mechanisms of Rheum $L$. in the treatment of diabetic kidney disease using a systems pharmacology approach.

\section{Methods}

A network pharmacology-based strategy was proposed to elucidate the underlying multi-component, multi-target, and multi-pathway mode of action of Rheum L. against diabetic kidney disease. We collected putative targets of Rheum $L$. and a network of the interactions among the putative targets of Rheum $L$. and known therapeutic targets of diabetic kidney disease was built. The major hubs were imported to the Database to perform a pathway enrichment analysis.

\section{Results}

A total of 6 active ingredients and 271 targets of Rheum L. were picked out. 11 cellular biological processes and 18 pathways of Rheum L. mostly associated with inflammatory response, apoptosis, fibrosis, and peripheral circulation.

\section{Conclusions}

Rheum L. could alleviate diabetic kidney disease via the molecular mechanisms predicted by network pharmacology.

\section{Background}

Diabetic kidney disease (DKD) is one of the most common and serious complications of diabetes mellitus. Pathological changes in renal cells of DKD patients include glomerular hypertrophy, mesangial expansion, and tubulointerstitial fibrosis due to the accumulation of extracellular matrix (ECM) proteins, thickening of basement membrane, and podocyte dysfunction ${ }^{1}$. DKD is now the main cause of CKD worldwide and the leading cause of end-stage-renal disease (ESRD) requiring renal replacement therapy. Approximately $20-40 \%$ of diabetic patients suffer from DKD in China, which is the main cause of CKD and end-stage renal failure in patients with diabetes ${ }^{2}$. DKD is a major but under-recognized contributor to the global burden of disease. Between 1990 and 2012, the number of deaths attributed to DKD rose by $94 \%{ }^{3}$. This dramatic rise is one of the highest observed for all reported chronic diseases ${ }^{4}$. Early medical research has shown that angiotensin-converting enzyme inhibitors (ACEls) and angiotensin II receptor blockers (ARBs), the first-line treatment for DKD, can reduce proteinuria and have a certain effect on 
delaying the progress of renal dysfunction ${ }^{5}$. However, the side effects of ACEI/ARB, such as dry cough, hypotension, hyperkalemia, and angioedema, limit the application of these drugs. Evidence-based medical studies have shown that these agents have not significantly reduced DKD vascular event rate and mortality ${ }^{6}$. Therefore, it is urgent to discover potential therapeutic targets and develop new therapeutic strategies for the treatment of DKD.

Traditional Chinese Medicine (TCM) is a branch of traditional oriental medicine, characterized by its systematic and holistic philosophy, which has been widely used in Asian countries ${ }^{7}$. TCM have multiple ingredients that contain a variety of advantages, such as synergy, minor side effects, and improvement of adaptive resistance ${ }^{8}$. TCM network pharmacology not only confirms and optimizes multiple targets interventions by modeling signaling pathways and precise processes, but also estimates the efficacy of drugs, especially multi-target drugs ${ }^{9,10}$. Based on the theory of traditional Chinese herbal medical science, TCM can offer a treatment for the prevention and treatment of DKD in a systematic way. As a traditional medicinal plant, Rheum $L$. has satisfactory drug efficacy for the alleviation of DKD, which indicates the existence of certain pharmacological components in Rheum $L .{ }^{11}$. In clinical practice, we discovered that some formulas, which contained Rheum L., could effectively decrease 24hurinary protein level, increase glomerular filtration rate in type 2 DKD patients with macroalbuminuria, and ameliorate diabetic kidney injury ${ }^{12,13}$. However, the pharmacological mechanisms of Rheum $L$. are still vague.

The aim of our study was to screen the related ingredients of Rheum $L$. using multiple databases and acquire the potential targets by target fishing. Then, we aimed to screen the related targets of DKD by consolidation of a multi-source database. Based on the matching results between Rheum L. potential targets and DKD targets, we aimed to build a protein-protein interaction (PPI) network to analyze the interactions among these targets and screen the hub targets based on topology. Moreover, using The DAVID bioinformatics resources, we aimed to obtain the enrichment analysis of the GO-BP and KEGG. This study is necessary to investigate how Rheum L. alleviates DKD via the molecular mechanisms predicted by network pharmacology and how the network pharmacology approach can be an effective tool to reveal the mechanisms of TCM. The flowchart of the experimental procedures of our study is shown in Fig. 1.

\section{Methods}

2.1 Ingredients in Rheum L. and its targets

To collect the ingredients in Rheum L., we used the Traditional Chinese Medicine System Pharmacology Database ${ }^{14}$ (TCMSP, http://lsp.nwu.edu.cn/tcmsp.php, 2020.2.28). Ninety-two herbal ingredients were recorded in this process. Retrieving the small molecular structure information of the active ingredients in Rheum L. on the PubChem database (https://pubchem.ncbi.nlm.nih.gov/) and the Swiss Target Prediction webserver (http://www.http:// www.swisstargetprediction.ch/index.php).

2.2 Disease targets database building 
We collected DKD targets from four source databases. The databases used in our study were: the DrugBank database (http://www.drugbank.ca/, version 4.3, 2020.2.28), Online Mendelian Inheritance in Man (OMIM) database ${ }^{15}$ (http://www.omim.org/, 2020.2.28), Therapeutic Target Database (http://db.idrblab.org/ttd, 2020.2.28), and Genetic Association Database ${ }^{16}$ (https://www.geneticassociationdb.nih.gov/, 2020.2.28). The targets were then processed by String (https://string-db.org/, 2020.3.3) to draw the data of PPI.

\subsection{Network construction}

1. Compound-target network (C-T network); 2. Rheum L. target-DKD target interactional network (T-T network); 3. Target-pathway network (T-P network). The pathway information of targets was screened from the result of KEGG pathway enrichment. Cytoscape3.6.0 (http://www.cytoscape.org/, 2020.3.3).

\subsection{Enrichment analysis}

We used DAVID ${ }^{17}$ (https://david.ncifcrf.gov/, v6.8,2020.3.3) for GO enrichment analysis and KEGG ${ }^{18}$ (http://www.kegg.jp/,2020.3.3) for pathway enrichment analysis.

\section{Results}

\subsection{Active compounds in Rheum L.}

Retrieved from TCMSP, there were 92 related components in the whole formula in total. According to the active ingredient screening thresholds of $O B \geq 30 \%$ and $D L \geq 0.18,16$ active ingredients were selected.

\subsection{Target prediction and analysis}

We conducted target fishing on the 16 active ingredients based on chemical similarity, obtaining 271 related targets. The 16 active compounds that were obtained are listed in Table 1. 
Table 1

The list of active ingredients of Rheum $L$.

\begin{tabular}{|llll|}
\hline ID & Compound & OB (\%) & DL \\
\hline MOL002235 & EUPATIN & 50.8 & 0.41 \\
\hline MOL002251 & Mutatochrome & 48.64 & 0.61 \\
\hline MOL002259 & Physciondiglucoside & 41.65 & 0.63 \\
\hline MOL002260 & Procyanidin B-5,3'-O-gallate & 31.99 & 0.32 \\
\hline MOL002268 & rhein & 47.07 & 0.28 \\
\hline MOL002276 & Sennoside E_qt & 50.69 & 0.61 \\
\hline MOL002280 & Torachrysone-8-O-beta-D-(6'-oxayl)-glucoside & 43.02 & 0.74 \\
\hline MOL002281 & Toralactone & 46.46 & 0.24 \\
\hline MOL002288 & Emodin-1-O-beta-D-glucopyranoside & 44.81 & 0.80 \\
\hline MOL002293 & Sennoside D_qt & 61.06 & 0.61 \\
\hline MOL002297 & Daucosterol_qt & 35.89 & 0.70 \\
\hline MOL002303 & palmidin A & 32.45 & 0.65 \\
\hline MOL000358 & beta-sitosterol & 36.91 & 0.75 \\
\hline MOL000471 & aloe-emodin & 83.38 & 0.24 \\
\hline MOL000554 & gallic acid-3-O-(6'-O-galloyl)-glucoside & 30.25 & 0.67 \\
\hline MOL000096 & (-)-catechin & 49.68 & 0.24 \\
\hline
\end{tabular}

Twenty three targets that matched the related targets of Rheum $L$. on DKD were collected as related targets for the effect of Rheum L. on DKD (Fig. 2 and Fig. 3).

\subsection{GO biological process and KEGG pathway enrichment analysis}

In the String database, the PPI network of the 23 targets was established. The details are shown in Fig. 4. There were 23 nodes and 65 edges in total. The threshold values were degree $\geq 5.9$ and closeness $\geq 0.51$ and the results settled at 23 hub nodes and 65 edges. The details are shown in Fig. 5, which includes Caspase-3 (CASP3) (degree = 14), Cyclooxygenase-2 (PTGS2) (degree = 12)Angiotensin-converting enzyme(ACE) (degree = 11), Peroxisome proliferator-activated receptor gamma (PPARG) $($ degree $=9)$, Insulin-like growth factor I receptor (IGF1R) (degree = 9), Glycogen synthase kinase-3 beta (GSK3B) (degree = 8), Beta amyloid A4 protein (APP) (degree = 8), Androgen Receptor (AR) (degree = 7), Thrombin (F2) (degree = 6), Plasminogen activator inhibitor-1 (SERPINE1) (degree = 6), Hepatocyte growth factor receptor $(\mathrm{MET})($ degree $=6)$, and Hepatocyte nuclear factor 4-alpha $(H N F 4 A)($ degree $=6)$. 
represent the degree, as the scale indicates. The size of the circle also indicates the degree.

\subsubsection{GO biological process enrichment analysis}

DAVID v6.8 was used for enrichment analysis of the 23 targets. The screening threshold was $\mathrm{P}<0.01$ and $11 \mathrm{GO}$ items were retrieved. We selected 18 KEGG pathways for analysis. The details are shown in Fig. 6.

Figure 6. The main 18 pathways enriched by major hubs from the DAVID database.

\subsubsection{KEGG pathway enrichment analysis}

The 11 biological processes were mainly involved in inflammatory response, apoptosis, fibrosis, and peripheral circulation. The details are shown in Fig. 7. The processes were, in the aspect of cell proliferation: positive regulation of transcription from RNA polymerase II promoter (GO:0045944), positive regulation of cell proliferation (G0:0008284), transmembrane receptor protein tyrosine kinase signaling pathway (G0:0007169), and transcription, DNA-templated (G0:0006351);in the aspect of protein metabolism: negative regulation of protein binding (G0:0032091) and positive regulation of protein binding (G0:0032092); in the aspect of inflammatory response: response to oxidative stress (G0:0006979); in the aspect of apoptosis: negative regulation of extrinsic apoptotic signaling pathway (G0:2001237); and in the aspect of peripheral circulation regulation: glucose homeostasis(G0:0042593), regulation of blood pressure(G0:0008217), and blood coagulation(GO:0007596). Based on these five main aspects, a complex multi-path synergetic effect may be the cause of the effect of Rheum L. on DKD.

\section{Discussion}

\subsection{Inflammatory response}

It is known that PTGS2 is often called cyclooxygenase 2 (COX-2), several inflammatory cytokines contribute to DKD pathogenesis, including COX-2, inflammatory parameters in patients with type 2 diabetes at an early stage of nephropathy are independently associated with urinary albumin excretion $(\mathrm{UAE})^{19}$. High glucose activates protein kinase $\mathrm{C}$ (PKC) to increase the permeability of Glomerular endothelial cells (GECs), which up-regulates the COX-2 in endothelial cells $\square$ and COX-2 up-regulation is associated with an imbalance in eicosanoids profile, with an increase in vasoconstricting thromboxane $\mathrm{B} 2$ and a decrease in vasodilatory 6-keto-prostaglandin $\mathrm{Fa}^{20}$. These changes lead to renal hemodynamic changes, which may participate in glomerular hyperfiltration observed in early DKD. In addition, increased ROS production upregulates COX-2 gene transcription and modifies the production of prostanoids ${ }^{21}$,

${ }^{22}$ Wwich control vascular reactivity of the renal glomerular arterioles and deteriorate the glomerular endothelial surface layer ${ }^{23}$.In mice, diabetes-induced endothelial injury, albuminuria, podocyte loss, and glomerulosclerosis are ameliorated by scavenging of mitochondrial ROS prevent endothelial mitochondrial oxidative stress ${ }^{24}$. 
Adiponectin exerts favorable effects in anti-inflammatory, antifibrotic, antioxidant effects, and potently stimulates ceramidase activity. Low circulating adiponectin levels in obese patients who have a risk of insulin resistance, type 2 diabetes, and cardiovascular diseases, and increased adiponectin expression in the state of albuminuria suggest a protective and compensatory role for adiponectin in mitigating further renal injury during the development of overt DKD. Enhancing the AMPK/PPARa pathway and ceramidase activity alleviate renoprotective role against lipotoxicity and oxidative stress ${ }^{25}$. Moreover, a study demonstrated that peroxisome proliferator-activated receptor y (PPARY) coactivator-1a (PGC-1a) participates in mitochondrial biogenesis in tissues with high energy consumption, as a key nuclear factor in energy and oxidative stress ${ }^{26}$. PGC- $1 a$ is highly expressed in the kidney ${ }^{27}$, and is reported to protect against several kidney diseases. PGC-1 a upregulation has been demonstrated to alleviate mitochondrial dysfunction in acute kidney injury and an adriamycin nephrosis model 28,29 .

IGF1 is a powerful regulatory factor in various cell types, including glomerular and tubular cells ${ }^{30}$. It is an important growth factor for keeping the nephritic structure and function. It also plays a key role in the pathological process of DKD ${ }^{31}$. Inhibition of IGF1R could alleviate inflammation in DKD more efficiently ${ }^{32}$. Li J el al ${ }^{33}$ found that inhibition of IGF1R was a more effective choice for inflammation treatment than insulin in diabetic kidney disease (DKD). The IGF1R inhibitor blocked pathological changes induced by the over-expression of IGF1 in DKD without up-regulating SOCS2 protein levels.

\subsection{Apoptosis}

Apoptosis, which programmed cell death, plays an essential role in the development and homeostasis of metazoans. Deregulation of apoptosis leads to a variety of pathologic disorders, including cancer, autoimmune diseases, and neurodegenerative disorders. Caspases, part of the cysteine protease family, are central to the initiation and execution of apoptosis, acting to specifically cleave the C-terminal side of an aspartate residue in substrates. The apoptotic caspases are generally classified into initiator and executioner caspases,and one of the principal executioner caspases is Caspase- $3^{34,35}$. Researchers have found that apoptosis had an indispensable role in renal ischemia-reperfusion injury and contrast-induced acute kidney injury ${ }^{36,37}$. Markers of apoptosis have repeatedly been linked with DKD ${ }^{38}$. Wang et al ${ }^{39}$ showed that GSDME could switch Caspase-3-dependent non-inflammatory and immunologically silent apoptosis to a terminal phase, namely secondary necrosis. Wen et al ${ }^{40}$ found that Caspase- 3 could promote renal injury and fibrosis in DKD through gasdermin E-mediated progression to secondary necrosis during apoptosis.

IGF-I is synthesized in renal glomeruli and distal tubules. IGF-I raises GFR through reducing arteriolar resistance and increasing LPA. IGF-I has been associated with the initiation of hypertrophy in models of compensatory renal growth and may contribute to the accumulation of extracellular matrix proteins in the nephron in chronic renal diseases. IGF-1 can promote sclerosis by either increasing synthesis, or decreasing the degradation of glomerular extracellular matrix (ECM), suggesting that IGF-1 could be a major contributor to the development of diabetic glomerulosclerosis ${ }^{41,42}$. In addition, insulin and IGF-1 
are major anti-apoptotic hormones, as well as metabolic and growth hormones. Their effects are mediated through activation of the insulin and IGF-1R and the consequent activation of the PI3K-Akt (phosphatidylinositol 3-kinase to Akt) and the Ras-Raf-MEK-ERK (Ras to Raf to mitogen-activated or extracellular signal-regulated protein kinase to extracellular signal-regulated protein kinase) pathways 43 , ${ }^{44}$.In brown preadipocytes in the basal state, the presence of insulin or IGF-1 receptors plays a permissive role in apoptosis, which is blocked either by ligand binding or by deletion of both receptors, revealing the apoptosis that is independent of their kinase activity ${ }^{45}$.

\subsection{Fibrosis}

PPARy belongs to the ligand-activated type II nuclear receptor superfamily and predominantly expressed in adipose tissues ${ }^{46}$. In the kidney, PPARY is mainly located in the medullary collecting duct, and glomeruli and proximal tubules were also expressed in small amounts ${ }^{47}$. Activated PPARy delayed the progress of DKD by improving insulin resistance ${ }^{48}$, lowering blood pressure ${ }^{49}$, ameliorating inflammation ${ }^{50}$, reversing cell cycle arrest ${ }^{51}$, increasing adiponectin ${ }^{52}$, improving oxidative stress ${ }^{53}$, and other mechanisms. On the other hand, the study has shown that PPARY single nucleotide polymorphism (SNP) is associated with the risk of diabetic kidney disease ${ }^{54}$. All of these indicated that PPARy signal pathway plays a protective role in DKD ${ }^{55}$. Moreover, The PPARy agonist may induce PGC-1 a expression to maintain mitochondrial function and to reduce ROS generation, and it may ameliorate podocyte impairment, GBM thickening and kidney fibrosis to aid in the prevention of DKD occurrence and progression ${ }^{56}$.

IGF-1 participates in multiple biological metabolic pathways of DKD. Dong et al ${ }^{57}$ discovered that IGF-1R inhibitor could ameliorate urinary albumin excretion and kidney histological changes due to diabetes, including amelioration of glomerulomegaly, inflammatory infiltration, and tubulointerstitial fibrosis. Activation of IGF-1 in diabetic kidneys induces fibrogenesis through Snail1 upregulation. The diabetesrelated histological and functional changes, as well as fibrogenesis, can be attenuated by IGF-1/IGF-1R inhibition.

\subsection{Peripheral circulation regulation}

In type 1 diabetes (T1D), adjuvant treatment with inhibitors of the renin-angiotensin-aldosterone system (RAAS), which dilate the efferent arteriole, is associated with prevention of progressive albuminuria and renal dysfunction. In the setting of chronic hyperglycemia, overactivation of the RAAS is strongly implicated in the initiation and progression of DKD ${ }^{58,59}$. Angiotensin II (Ang II) is the major peptide of RAS, and is formed under the action of the angiotensin converting enzyme (ACE). Ang II shows increased activity under high glucose conditions that causes hypertrophy of various renal cells and has a pressor effect on arteriolar smooth muscles, thereby resulting in increased vascular pressure ${ }^{60}$. The ACE gene is composed of 26 exons and 25 introns, with a length of $21 \mathrm{~kb}$. Recently, many researchers have demonstrated that the ACE I/D polymorphism is related to diabetic microangiopathy, and that the $D$ allele 
might be a susceptibility factor for patients with DKD ${ }^{61-63}$. The ACE I/D polymorphism was suggested to be strongly associated with the development of DKD, and using valsartan in DKD patients could significantly decrease the excretion of urinary albumin ${ }^{64}$.

\subsection{Rheum L.}

Rheum L., one of the most popular traditional Chinese medicines used to control various diseases for thousands of years, there are several studies that demonstrate that Rheum $L$. can effectively reduce inflammation, apoptosis, antioxidant stress, and blood pressure.

\subsubsection{Glycolipid metabolism}

Cheng et al. ${ }^{65}$ found that the fasting blood glucose (FBG), total cholesterol (TC), and triglyceride (TG) levels in the serum of rats in the purified anthraquinone-Glycoside from Rheum palmatum $L$. treatment groups were significantly decreased. The expression of Fas ligand (FasL), cytochrome C (Cyt-c), and Caspase-3 in pancreatic tissue was obviously decreased, and the pathological damage to the liver, kidney, and pancreas was improved, which indicated that PAGR can reduce oxidative stress in rats with diabetes mellitus by improving blood lipid metabolism and enhancing their antioxidant capacity, thereby regulating the mitochondrial apoptotic pathway to inhibit $\beta$-cell apoptosis and improve $\beta$-cell function. Furthermore, PAGR could activate the GLP-1/cAMP pathway to decrease glycated serum protein (GSP), insulin concentration and HOMA-IR index, increase GLP-1 concentrations, ameliorate insulin resistance, and restore the gut microbiota ${ }^{66}$.

\subsubsection{Improving renal function}

The changes in mean blood glucose levels, normalized kidney weight, urinary albumin excretion, serum creatinine levels and tubulointerstitial injury index (TII) scores of the rats with DN were significantly attenuated by emodin. Furthermore, treatment with emodin significantly inhibited inflammation-related factors and oxidative stress, suppressed the expression of intercellular adhesion molecule 1 (ICAM-1) and B-cell lymphoma 2-associated X protein (Bax), increased phosphorylated Akt and phosphorylatedglycogen synthase kinase 3 ( $p-G S K-3 \beta$ ) expression and inhibited caspase-3 activity in diabetic rats ${ }^{67}$. Li et al ${ }^{68}$ discovered that using $R$ heum $L$. anthraquinones extract decreased the serum creatinine ( $\mathrm{SCr}$ ), blood urea nitrogen (BUN) and urine protein (UP) values in diabetic nephropathy rats, which suggested that it displayed certain therapeutic and preventive effects against the diabetic nephropathy. Moreover, administration of Rheum L. inhibited increase of BUN and serum creatinine in cases with chronic renal failure and was effective for the reduction of uremic substances, which can retard the introduction-period of hemodialysis and inhibit deterioration of the disease ${ }^{69}$. Gentamicin increased the level of urinary glucose and protein, and increased malondialdehyde while it decreased thiol in kidney tissue, and increased the concentration of urea and creatinine in the serum. However, hydroalcoholic extract of Rheum turkestanicum was able to improve gentamicin toxicity, it has positive effects in the attenuation of gentamicin-induced nephrotoxicity ${ }^{70}$. 


\subsubsection{Delaying renal fibrosis}

Shui et al ${ }^{71}$ found that Dahuang Fuzi Decoction could attenuate renal fibrosis and ameliorates mitochondrial dysfunction in chronic aristolochic acid nephropathy. Besides, Dahuang Fuzi Decoction could alleviate adenine-induced tubular epithelial apoptosis and renal damage in vivo, presumably through the suppression of TGF- $\beta 1$-JNK pathway activation ${ }^{72}$. Chinese researchers believed that Niaoduqing particles safely and effectively delayed CKD progression in patients with stage 3b-4 CKD. This traditional Chinese medicine may be a promising alternative medication for patients with moderateto-severe renal dysfunction ${ }^{73}$. As the main active ingredient of Rheum L., emodin regulates lipopolysaccharide-induced toll-like receptor 4 and reduces the expression of tumor necrosis factor alpha and interleukin 6 , all three being synthesized by renal tubular epithelial cells ${ }^{74}$. Emodin acts as an antiinflammatory agent by inhibiting the differentiation and maturation of dendritic cells and increasing the number of regulatory T-cells ${ }^{75}$. It also can bring high blood pressure down, decrease blood lipids and improve microcirculation, protect kidney ${ }^{76}$. Tu et al ${ }^{77}$ discovered that rhein could inhibit autophagy in rat renal tubular cells by the regulation of the AMPK/mTOR, p38/Erk MAPKs and Akt-independent signaling pathways, which may explain the therapeutic mechanisms of Rheum L. and rhein in treating CKD patients.

Advances in traditional herbal medicine suggest Chinese herbal remedies can be considered promising agents in the prevention and treatment of DKD. However, the clinical application of Chinese herbal medications is pretty limited, due to the following concerns and barriers (1) Lacking of high-quality animal studies,focusing on the role of herbal medicine on DKD. (2) The potential side effects associated with herbal medicine may be underrecognized and underreported. (3) The screened active ingredients may be inconsistent with the actual absorbed components in the blood of patients with DKD. (4) The interaction relationships between the nodes in the network construction methods are still unclear. Therefore, further experimental verification of the potential active ingredients is needed to verify this theoretical prediction.

\section{Conclusion}

This study used a scientific approach to decipher the pharmacological mechanisms of Rheum L. in the treatment of DKD. We discovered that the effects may be associated with inflammatory response, apoptosis, fibrosis, and peripheral circulation. Among these crucial biological functions, twelve targets were identified as key active factors involved in the related pathways. This research suggests that Rheum L. can alleviate DKD via the molecular mechanisms predicted by network pharmacology and that the network pharmacology approach can be an effective tool to reveal the mechanisms of TCM. However, to improve the reliability of the results, further experimental experiments are needed to validate these results.

\section{Abbreviations}


DKD, diabetic kidney disease; TCM, traditional Chinese medicine; TCMSP, Traditional Chinese Medicine Systems Pharmacology Database; OB, Oral Bioavailability; DL, Drug-likeness; GO, Gene Ontology; KEGG, Kyoto Encyclopedia of Genes and Genomes; PPI, protein-protein interaction; DAVID, Database for Annotation, Visualization and Integrated Discovery; OMIM, Online Mendelian Inheritance in Man; TTD, Therapeutic Target Database; GAD, Genetic Association Database; C-T, compound-target; T-T, target-target; T-P, target-pathway.

\section{Declarations}

\section{Ethics approval and consent to participate}

Not applicable.

\section{Competing interests}

The authors declare that they have no competing interests.

\section{Availability of data and materials}

The data and materials generated or analyzed during this study are available from the corresponding author on reasonable request.

\section{Funding}

This work was supported by the 2015 Traditional Chinese Medicine Scientific Research (grant number: 201507001-11) and The National Natural Science Foundation of China (grant number: 81973813).

\section{Authors' Contributions}

Conceptualization, J.L., C.P. and D.J.; Methodology, D.J.; Software, C.P.; Validation, J.L.,C.P., D.J. and X.Z.; Formal Analysis, J.L. and C.P.; Investigation, L.W.; Resources, F.L. and X.T.; Data Curation, J.L.; WritingOriginal Draft Preparation, J.L.; Writing-Review \& Editing, C.P.; Visualization, J.L. and C.P.; Supervision, F.L. and X.T.; Project Administration, F.L. and X.T.; Funding Acquisition, F.L. and X.T.

\section{Acknowledgement}

Not applicable.

\section{Authors' information}

\section{Affiliations}

Institution of Shenzhen Hospital, Guangzhou University of Chinese Medicine (Futian), Shenzhen, 518000, Guangdong, China. 
Chun-Li Piao, Jin-Li Luo, Xiaohua Zhao囚Li Wang.

Institution of Guang'anmen Hospital, China Academy of Chinese Medical Science, Beijing, 100000, China.

De Jin, Feng-Lian Mei区Xiao-Lin Tong.

\section{Corresponding author}

Correspondence to Chun-Li Piao,Feng-Mei Lian囚Xiao-Lin Tong.

\section{References}

1. T.S. Assmann, M. Recamonde-Mendoza, B.M. de Souza, A.C. Bauer, D. Crispim, MicroRNAs and diabetic kidney disease: Systematic review and bioinformatic analysis, Molecular and cellular endocrinology 477 (2018) 90-102.

2. L. Zhang, J. Long, W. Jiang, Y. Shi, X. He, Z. Zhou, Y. Li, R.O. Yeung, J. Wang, K. Matsushita, J. Coresh, M.H. Zhao, H. Wang, Trends in Chronic Kidney Disease in China, The New England journal of medicine 375(9) (2016) 905-6.

3. R. Lozano, M. Naghavi, K. Foreman, S. Lim, K. Shibuya, V. Aboyans, J. Abraham, T. Adair, R. Aggarwal, S.Y. Ahn, M. Alvarado, H.R. Anderson, L.M. Anderson, K.G. Andrews, C. Atkinson, L.M. Baddour, S. BarkerCollo, D.H. Bartels, M.L. Bell, E.J. Benjamin, D. Bennett, K. Bhalla, B. Bikbov, A. Bin Abdulhak, G. Birbeck, F. Blyth, I. Bolliger, S. Boufous, C. Bucello, M. Burch, P. Burney, J. Carapetis, H. Chen, D. Chou, S.S. Chugh, L.E. Coffeng, S.D. Colan, S. Colquhoun, K.E. Colson, J. Condon, M.D. Connor, L.T. Cooper, M. Corriere, M. Cortinovis, K.C. de Vaccaro, W. Couser, B.C. Cowie, M.H. Criqui, M. Cross, K.C. Dabhadkar, N. Dahodwala, D. De Leo, L. Degenhardt, A. Delossantos, J. Denenberg, D.C. Des Jarlais, S.D. Dharmaratne, E.R. Dorsey, T. Driscoll, H. Duber, B. Ebel, P.J. Erwin, P. Espindola, M. Ezzati, V. Feigin, A.D. Flaxman, M.H. Forouzanfar, F.G. Fowkes, R. Franklin, M. Fransen, M.K. Freeman, S.E. Gabriel, E. Gakidou, F. Gaspari, R.F. Gillum, D. Gonzalez-Medina, Y.A. Halasa, D. Haring, J.E. Harrison, R. Havmoeller, R.J. Hay, B. Hoen, P.J. Hotez, D. Hoy, K.H. Jacobsen, S.L. James, R. Jasrasaria, S. Jayaraman, N. Johns, G. Karthikeyan, N. Kassebaum, A. Keren, J.P. Khoo, L.M. Knowlton, O. Kobusingye, A. Koranteng, R. Krishnamurthi, M. Lipnick, S.E. Lipshultz, S.L. Ohno, J. Mabweijano, M.F. MacIntyre, L. Mallinger, L. March, G.B. Marks, R. Marks, A. Matsumori, R. Matzopoulos, B.M. Mayosi, J.H. McAnulty, M.M. McDermott, J. McGrath, G.A. Mensah, T.R. Merriman, C. Michaud, M. Miller, T.R. Miller, C. Mock, A.O. Mocumbi, A.A. Mokdad, A. Moran, K. Mulholland, M.N. Nair, L. Naldi, K.M. Narayan, K. Nasseri, P. Norman, M. O'Donnell, S.B. Omer, K. Ortblad, R. Osborne, D. Ozgediz, B. Pahari, J.D. Pandian, A.P. Rivero, R.P. Padilla, F. Perez-Ruiz, N. Perico, D. Phillips, K. Pierce, C.A. Pope, 3rd, E. Porrini, F. Pourmalek, M. Raju, D. Ranganathan, J.T. Rehm, D.B. Rein, G. Remuzzi, F.P. Rivara, T. Roberts, F.R. De Leon, L.C. Rosenfeld, L. Rushton, R.L. Sacco, J.A. Salomon, U. Sampson, E. Sanman, D.C.

Schwebel, M. Segui-Gomez, D.S. Shepard, D. Singh, J. Singleton, K. Sliwa, E. Smith, A. Steer, J.A. Taylor, B. Thomas, I.M. Tleyjeh, J.A. Towbin, T. Truelsen, E.A. Undurraga, N. Venketasubramanian, L. Vijayakumar, T. Vos, G.R. Wagner, M. Wang, W. Wang, K. Watt, M.A. Weinstock, R. Weintraub, J.D. Wilkinson, A.D. Woolf, S. 
Wulf, P.H. Yeh, P. Yip, A. Zabetian, Z.J. Zheng, A.D. Lopez, C.J. Murray, M.A. AlMazroa, Z.A. Memish, Global and regional mortality from 235 causes of death for 20 age groups in 1990 and 2010: a systematic analysis for the Global Burden of Disease Study 2010, Lancet (London, England) 380(9859) (2012) 2095128.

4. Global, regional, and national burden of chronic kidney disease, 1990-2017: a systematic analysis for the Global Burden of Disease Study 2017, Lancet (London, England) 395(10225) (2020) 709-733.

5. D. Giugliano, M.I. Maiorino, G. Bellastella, K. Esposito, Comment on American Diabetes Association. Approaches to Glycemic Treatment. Sec. 7. In Standards of Medical Care in Diabetes-2016. Diabetes Care 2016;39(Suppl. 1):S52-S59, Diabetes care 39(6) (2016) e86-7.

6. E. Dounousi, A. Duni, K. Leivaditis, V. Vaios, T. Eleftheriadis, V. Liakopoulos, Improvements in the Management of Diabetic Nephropathy, The review of diabetic studies : RDS 12(1-2) (2015) 119-33.

7. S. Li, S. Xutian, New Development in Traditional Chinese Medicine: Symbolism-Digit Therapy as a Special Naturopathic Treatment, The American journal of Chinese medicine 44(7) (2016) 1311-1323.

8. S. Li, B. Zhang, N. Zhang, Network target for screening synergistic drug combinations with application to traditional Chinese medicine, BMC systems biology 5 Suppl 1 (2011) S10.

9. J.B. Fitzgerald, B. Schoeberl, U.B. Nielsen, P.K. Sorger, Systems biology and combination therapy in the quest for clinical efficacy, Nature chemical biology 2(9) (2006) 458-66.

10. P. Csermely, V. Agoston, S. Pongor, The efficiency of multi-target drugs: the network approach might help drug design, Trends in pharmacological sciences 26(4) (2005) 178-82.

11. Z. Lu, Y. Zhong, W. Liu, L. Xiang, Y. Deng, The Efficacy and Mechanism of Chinese Herbal Medicine on Diabetic Kidney Disease, Journal of diabetes research 2019 (2019) 2697672.

12. P. Li, Y. Chen, J. Liu, J. Hong, Y. Deng, F. Yang, X. Jin, J. Gao, J. Li, H. Fang, G. Liu, L. Shi, J. Du, Y. Li, M. Yan, Y. Wen, W. Yang, Efficacy and safety of tangshen formula on patients with type 2 diabetic kidney disease: a multicenter double-blinded randomized placebo-controlled trial, PloS one 10(5) (2015) e0126027.

13. J. Lv, Z. Wang, Y. Wang, W. Sun, J. Zhou, M. Wang, W.J. Liu, Y. Wang, Renoprotective Effect of the Shen-Yan-Fang-Shuai Formula by Inhibiting TNF-alpha/NF-kappaB Signaling Pathway in Diabetic Rats, Journal of diabetes research 2017 (2017) 4319057.

14. J. Ru, P. Li, J. Wang, W. Zhou, B. Li, C. Huang, P. Li, Z. Guo, W. Tao, Y. Yang, X. Xu, Y. Li, Y. Wang, L. Yang, TCMSP: a database of systems pharmacology for drug discovery from herbal medicines, Journal of cheminformatics 6 (2014) 13. 
15. J.S. Amberger, A. Hamosh, Searching Online Mendelian Inheritance in Man (OMIM): A Knowledgebase of Human Genes and Genetic Phenotypes, Current protocols in bioinformatics 58 (2017) 1.2.1-1.2.12.

16. H. Wang, X. Liu, Y. Tao, W. Ye, Q. Jin, W.W. Cohen, E.P. Xing, Automatic Human-like Mining and Constructing Reliable Genetic Association Database with Deep Reinforcement Learning, Pacific Symposium on Biocomputing. Pacific Symposium on Biocomputing 24 (2019) 112-123.

17. W. Huang da, B.T. Sherman, R.A. Lempicki, Systematic and integrative analysis of large gene lists using DAVID bioinformatics resources, Nature protocols 4(1) (2009) 44-57.

18. L. Chen, Y.H. Zhang, S. Wang, Y. Zhang, T. Huang, Y.D. Cai, Prediction and analysis of essential genes using the enrichments of gene ontology and KEGG pathways, PloS one 12(9) (2017) e0184129.

19. J.F. Navarro, C. Mora, M. Maca, J. Garca, Inflammatory parameters are independently associated with urinary albumin in type 2 diabetes mellitus, American journal of kidney diseases : the official journal of the National Kidney Foundation 42(1) (2003) 53-61.

20. F. Cosentino, M. Eto, P. De Paolis, B. van der Loo, M. Bachschmid, V. Ullrich, A. Kouroedov, C. Delli Gatti, H. Joch, M. Volpe, T.F. Luscher, High glucose causes upregulation of cyclooxygenase-2 and alters prostanoid profile in human endothelial cells: role of protein kinase $\mathrm{C}$ and reactive oxygen species, Circulation 107(7) (2003) 1017-23.

21. S. Prabhakar, J. Starnes, S. Shi, B. Lonis, R. Tran, Diabetic nephropathy is associated with oxidative stress and decreased renal nitric oxide production, Journal of the American Society of Nephrology : JASN 18(11) (2007) 2945-52.

22. E.A. Jaimes, P. Hua, R.X. Tian, L. Raij, Human glomerular endothelium: interplay among glucose, free fatty acids, angiotensin II, and oxidative stress, American journal of physiology. Renal physiology 298(1) (2010) F125-32.

23. A. Kuwabara, M. Satoh, N. Tomita, T. Sasaki, N. Kashihara, Deterioration of glomerular endothelial surface layer induced by oxidative stress is implicated in altered permeability of macromolecules in Zucker fatty rats, Diabetologia 53(9) (2010) 2056-65.

24. H. Qi, G. Casalena, S. Shi, L. Yu, K. Ebefors, Y. Sun, W. Zhang, V. D'Agati, D. Schlondorff, B. Haraldsson, E. Bottinger, I. Daehn, Glomerular Endothelial Mitochondrial Dysfunction Is Essential and Characteristic of Diabetic Kidney Disease Susceptibility, Diabetes 66(3) (2017) 763-778.

25. Y. Kim, C.W. Park, Mechanisms of Adiponectin Action: Implication of Adiponectin Receptor Agonism in Diabetic Kidney Disease, International journal of molecular sciences 20(7) (2019).

26. C. Liu, J.D. Lin, PGC-1 coactivators in the control of energy metabolism, Acta biochimica et biophysica Sinica 43(4) (2011) 248-57. 
27. C. Handschin, B.M. Spiegelman, Peroxisome proliferator-activated receptor gamma coactivator 1 coactivators, energy homeostasis, and metabolism, Endocrine reviews 27(7) (2006) 728-35.

28. M. Tran, S.M. Parikh, Mitochondrial biogenesis in the acutely injured kidney, Nephron. Clinical practice 127(1-4) (2014) 42-5.

29. F.B. Hickey, J.B. Corcoran, N.G. Docherty, B. Griffin, U. Bhreathnach, F. Furlong, F. Martin, C. Godson, M. Murphy, IHG-1 promotes mitochondrial biogenesis by stabilizing PGC-1alpha, Journal of the American Society of Nephrology : JASN 22(8) (2011) 1475-85.

30. B. Nambam, D. Schatz, Growth hormone and insulin-like growth factor-I axis in type 1 diabetes, Growth hormone \& IGF research : official journal of the Growth Hormone Research Society and the International IGF Research Society 38 (2018) 49-52.

31. L.A. Bach, L.J. Hale, Insulin-like growth factors and kidney disease, American journal of kidney diseases : the official journal of the National Kidney Foundation 65(2) (2015) 327-36.

32. M.Y. Donath, Multiple benefits of targeting inflammation in the treatment of type 2 diabetes, Diabetologia 59(4) (2016) 679-82.

33. J. Li, R. Dong, J. Yu, S. Yi, J. Da, F. Yu, Y. Zha, Inhibitor of IGF1 receptor alleviates the inflammation process in the diabetic kidney mouse model without activating SOCS2, Drug design, development and therapy 12 (2018) 2887-2896.

34. J.C. Timmer, G.S. Salvesen, Caspase substrates, Cell death and differentiation 14(1) (2007) 66-72.

35. S.J. Riedl, Y. Shi, Molecular mechanisms of caspase regulation during apoptosis, Nature reviews. Molecular cell biology 5(11) (2004) 897-907.

36. N. Miao, F. Yin, H. Xie, Y. Wang, Y. Xu, Y. Shen, D. Xu, J. Yin, B. Wang, Z. Zhou, Q. Cheng, P. Chen, H. Xue, L. Zhou, J. Liu, X. Wang, W. Zhang, L. Lu, The cleavage of gasdermin D by caspase-11 promotes tubular epithelial cell pyroptosis and urinary IL-18 excretion in acute kidney injury, Kidney international 96(5) (2019) 1105-1120.

37. Z. Zhang, X. Shao, N. Jiang, S. Mou, L. Gu, S. Li, Q. Lin, Y. He, M. Zhang, W. Zhou, Z. Ni, Caspase-11mediated tubular epithelial pyroptosis underlies contrast-induced acute kidney injury, Cell death \& disease 9(10) (2018) 983.

38. K. Susztak, A.C. Raff, M. Schiffer, E.P. Bottinger, Glucose-induced reactive oxygen species cause apoptosis of podocytes and podocyte depletion at the onset of diabetic nephropathy, Diabetes 55(1) (2006) 225-33.

39. Y. Wang, W. Gao, X. Shi, J. Ding, W. Liu, H. He, K. Wang, F. Shao, Chemotherapy drugs induce pyroptosis through caspase-3 cleavage of a gasdermin, Nature 547(7661) (2017) 99-103. 
40. S. Wen, Z.H. Wang, C.X. Zhang, Y. Yang, Q.L. Fan, Caspase-3 Promotes Diabetic Kidney Disease Through Gasdermin E-Mediated Progression to Secondary Necrosis During Apoptosis, Diabetes, metabolic syndrome and obesity : targets and therapy 13 (2020) 313-323.

41. E. Lupia, S.J. Elliot, O. Lenz, F. Zheng, M. Hattori, G.E. Striker, L.J. Striker, IGF-1 decreases collagen degradation in diabetic NOD mesangial cells: implications for diabetic nephropathy, Diabetes 48(8) (1999) 1638-44.

42. P. Catanuto, X. Xia, S. Pereira-Simon, S. Elliot, Estrogen receptor subtype ratio change protects against podocyte damage, Current trends in endocrinology 9 (2017) 19-29.

43. R. Kooijman, Regulation of apoptosis by insulin-like growth factor (IGF)-I, Cytokine \& growth factor reviews 17(4) (2006) 305-23.

44. R.T. Kurmasheva, P.J. Houghton, IGF-I mediated survival pathways in normal and malignant cells, Biochimica et biophysica acta 1766(1) (2006) 1-22.

45. J. Boucher, Y. Macotela, O. Bezy, M.A. Mori, K. Kriauciunas, C.R. Kahn, A kinase-independent role for unoccupied insulin and IGF-1 receptors in the control of apoptosis, Science signaling 3(151) (2010) ra87.

46. A. Chawla, E.J. Schwarz, D.D. Dimaculangan, M.A. Lazar, Peroxisome proliferator-activated receptor (PPAR) gamma: adipose-predominant expression and induction early in adipocyte differentiation, Endocrinology 135(2) (1994) 798-800.

47. T. Yang, D.E. Michele, J. Park, A.M. Smart, Z. Lin, F.C. Brosius, 3rd, J.B. Schnermann, J.P. Briggs, Expression of peroxisomal proliferator-activated receptors and retinoid $X$ receptors in the kidney, The American journal of physiology 277(6) (1999) F966-73.

48. S. Tiwari, V.K. Halagappa, S. Riazi, X. Hu, C.A. Ecelbarger, Reduced expression of insulin receptors in the kidneys of insulin-resistant rats, Journal of the American Society of Nephrology : JASN 18(10) (2007) 2661-71.

49. Q.N. Diep, M. El Mabrouk, J.S. Cohn, D. Endemann, F. Amiri, A. Virdis, M.F. Neves, E.L. Schiffrin, Structure, endothelial function, cell growth, and inflammation in blood vessels of angiotensin II-infused rats: role of peroxisome proliferator-activated receptor-gamma, Circulation 105(19) (2002) 2296-302.

50. S. Ohga, K. Shikata, K. Yozai, S. Okada, D. Ogawa, H. Usui, J. Wada, Y. Shikata, H. Makino, Thiazolidinedione ameliorates renal injury in experimental diabetic rats through anti-inflammatory effects mediated by inhibition of NF-kappaB activation, American journal of physiology. Renal physiology 292(4) (2007) F1141-50.

51. T. Okada, J. Wada, K. Hida, J. Eguchi, I. Hashimoto, M. Baba, A. Yasuhara, K. Shikata, H. Makino, Thiazolidinediones ameliorate diabetic nephropathy via cell cycle-dependent mechanisms, Diabetes 55(6) (2006) 1666-77.

Page 16/30 
52. Y. Miyazaki, E. Cersosimo, C. Triplitt, R.A. DeFronzo, Rosiglitazone decreases albuminuria in type 2 diabetic patients, Kidney international 72(11) (2007) 1367-73.

53. A. Gumieniczek, Effect of the new thiazolidinedione-pioglitazone on the development of oxidative stress in liver and kidney of diabetic rabbits, Life sciences 74(5) (2003) 553-62.

54. H. Zhang, S. Zhu, J. Chen, Y. Tang, H. Hu, V. Mohan, R. Venkatesan, J. Wang, H. Chen, Peroxisome proliferator-activated receptor gamma polymorphism Pro12Ala Is associated with nephropathy in type 2 diabetes: evidence from meta-analysis of 18 studies, Diabetes care 35(6) (2012) 1388-93.

55. X. Yang, X. Xiao, H. Wang, Y. Li, L. Wang, G. Li, S. Deng, Renoprotective Effect of Danhong Injection on Streptozotocin-Induced Diabetic Rats through a Peroxisome Proliferator-Activated Receptor gamma Mediated Pathway, Evidence-based complementary and alternative medicine : eCAM 2018 (2018) 3450141.

56. L. Zhang, J. Liu, F. Zhou, W. Wang, N. Chen, PGC-1alpha ameliorates kidney fibrosis in mice with diabetic kidney disease through an antioxidative mechanism, Molecular medicine reports 17(3) (2018) 4490-4498.

57. R. Dong, J. Yu, F. Yu, S. Yang, Q. Qian, Y. Zha, IGF-1/IGF-1R blockade ameliorates diabetic kidney disease through normalizing Snail1 expression in a mouse model, American journal of physiology. Endocrinology and metabolism 317(4) (2019) E686-e698.

58. D.Z. Cherney, J.W. Scholey, E. Sochett, T.J. Bradley, H.N. Reich, The acute effect of clamped hyperglycemia on the urinary excretion of inflammatory cytokines/chemokines in uncomplicated type 1 diabetes: a pilot study, Diabetes care 34(1) (2011) 177-80.

59. D.Z. Cherney, H.N. Reich, J.W. Scholey, D. Daneman, F.H. Mahmud, R.L. Har, E.B. Sochett, The effect of aliskiren on urinary cytokine/chemokine responses to clamped hyperglycaemia in type 1 diabetes, Diabetologia 56(10) (2013) 2308-17.

60. T. Chawla, D. Sharma, A. Singh, Role of the renin angiotensin system in diabetic nephropathy, World journal of diabetes 1(5) (2010) 141-5.

61. R. Shaikh, S.M. Shahid, Q. Mansoor, M. Ismail, A. Azhar, Genetic variants of ACE (Insertion/Deletion) and AGT (M268T) genes in patients with diabetes and nephropathy, Journal of the renin-angiotensinaldosterone system : JRAAS 15(2) (2014) 124-30.

62. Z. Rahimi, A. Vaisi-Raygani, Z. Rahimi, A. Parsian, Concomitant presence of endothelial nitric oxide 894T and angiotensin II-converting enzyme D alleles are associated with diabetic nephropathy in a Kurdish population from Western Iran, Nephrology (Carlton, Vic.) 17(2) (2012) 175-81.

63. R. El-Baz, A. Settin, A. Ismaeel, A.A. Khaleel, T. Abbas, W. Tolba, W. Abd Allah, M.A. Sobh, MTHFR C677T, A1298C and ACE I/D polymorphisms as risk factors for diabetic nephropathy among type 2 
diabetic patients, Journal of the renin-angiotensin-aldosterone system : JRAAS 13(4) (2012) 472-7.

64. Y. Wang, W. Peng, X. Zhang, H. Qiao, L. Wang, Z. Xu, C. Wu, The association of ACE gene polymorphism with diabetic kidney disease and renoprotective efficacy of valsartan, Journal of the reninangiotensin-aldosterone system : JRAAS 17(3) (2016).

65. F.R. Cheng, H.X. Cui, J.L. Fang, K. Yuan, Y. Guo, Ameliorative Effect and Mechanism of the Purified Anthraquinone-Glycoside Preparation from Rheum Palmatum L. on Type 2 Diabetes Mellitus, Molecules (Basel, Switzerland) 24(8) (2019).

66. H.X. Cui, L.S. Zhang, Y. Luo, K. Yuan, Z.Y. Huang, Y. Guo, A Purified Anthraquinone-Glycoside Preparation From Rhubarb Ameliorates Type 2 Diabetes Mellitus by Modulating the Gut Microbiota and Reducing Inflammation, Frontiers in microbiology 10 (2019) 1423.

67. D. Jing, H. Bai, S. Yin, Renoprotective effects of emodin against diabetic nephropathy in rat models are mediated via PI3K/Akt/GSK-3beta and Bax/caspase-3 signaling pathways, Experimental and therapeutic medicine 14(5) (2017) 5163-5169.

68. P. Li, Q. Lu, W. Jiang, X. Pei, Y. Sun, H. Hao, K. Hao, Pharmacokinetics and pharmacodynamics of rhubarb anthraquinones extract in normal and disease rats, Biomedicine $\&$ pharmacotherapy $=$ Biomedecine \& pharmacotherapie 91 (2017) 425-435.

69. H. Sanada, [Study on the clinical effect of rhubarb on nitrogen-metabolism abnormality due to chronic renal failure and its mechanism], Nihon Jinzo Gakkai shi 38(8) (1996) 379-87.

70. M.T. Boroushaki, S. Fanoudi, H. Mollazadeh, S. Boroumand-Noughabi, A. Hosseini, Reno-protective effect of Rheum turkestanicum against gentamicin-induced nephrotoxicity, Iranian journal of basic medical sciences 22(3) (2019) 328-333.

71. G.X. Shui, D. Sang, X. Yin, Y. Cai, W. Sun, Dahuang Fuzi Decoction Attenuates Renal Fibrosis and Ameliorates Mitochondrial Dysfunction in Chronic Aristolochic Acid Nephropathy, Evidence-based complementary and alternative medicine : eCAM 2017 (2017) 9536458.

72. Y. Tu, W. Sun, Y.G. Wan, K. Gao, H. Liu, B.Y. Yu, H. Hu, Y.R. Huang, Dahuang Fuzi Decoction ameliorates tubular epithelial apoptosis and renal damage via inhibiting TGF-beta1-JNK signaling pathway activation in vivo, Journal of ethnopharmacology 156 (2014) 115-24.

73. Y. Zheng, G.Y. Cai, L.Q. He, H.L. Lin, X.H. Cheng, N.S. Wang, G.H. Jian, X.S. Liu, Y.N. Liu, Z.H. Ni, J.A. Fang, H.L. Ding, W. Guo, Y.N. He, L.H. Wang, Y.P. Wang, H.T. Yang, Z.M. Ye, R.H. Yu, L.J. Zhao, W.H. Zhou, W.G. Li, H.J. Mao, Y.L. Zhan, Z. Hu, C. Yao, R.B. Wei, X.M. Chen, Efficacy and Safety of Niaoduqing Particles for Delaying Moderate-to-severe Renal Dysfunction: A Randomized, Double-blind, Placebocontrolled, Multicenter Clinical Study, Chinese medical journal 130(20) (2017) 2402-2409. 
74. X.L. Zhu, Y.J. Wang, Y. Yang, R.C. Yang, B. Zhu, Y. Zhang, Y. Lin, Y. Lu, X.F. Li, K.T. O'Byrne, Suppression of lipopolysaccharide-induced upregulation of toll-like receptor 4 by emodin in mouse proximal tubular epithelial cells, Molecular medicine reports 6(3) (2012) 493-500.

75. W. Zhang, H. Li, H. Bu, H. Chen, H. Tong, D. Liu, H. Guo, S.Z. Lin, Emodin inhibits the differentiation and maturation of dendritic cells and increases the production of regulatory $T$ cells, International journal of molecular medicine 29(2) (2012) 159-64.

76. S. Xiong, S. Lei, Simultaneous Determination of Five Active Components in the Chinese Patent Medicine Niuhuang Jiangya Pill by HPLC-MS/MS, Journal of AOAC International 100(3) (2017) 717-720.

77. Y. Tu, L. Gu, D. Chen, W. Wu, H. Liu, H. Hu, Y. Wan, W. Sun, Rhein Inhibits Autophagy in Rat Renal Tubular Cells by Regulation of AMPK/mTOR Signaling, Scientific reports 7 (2017) 43790.

\section{Figures}




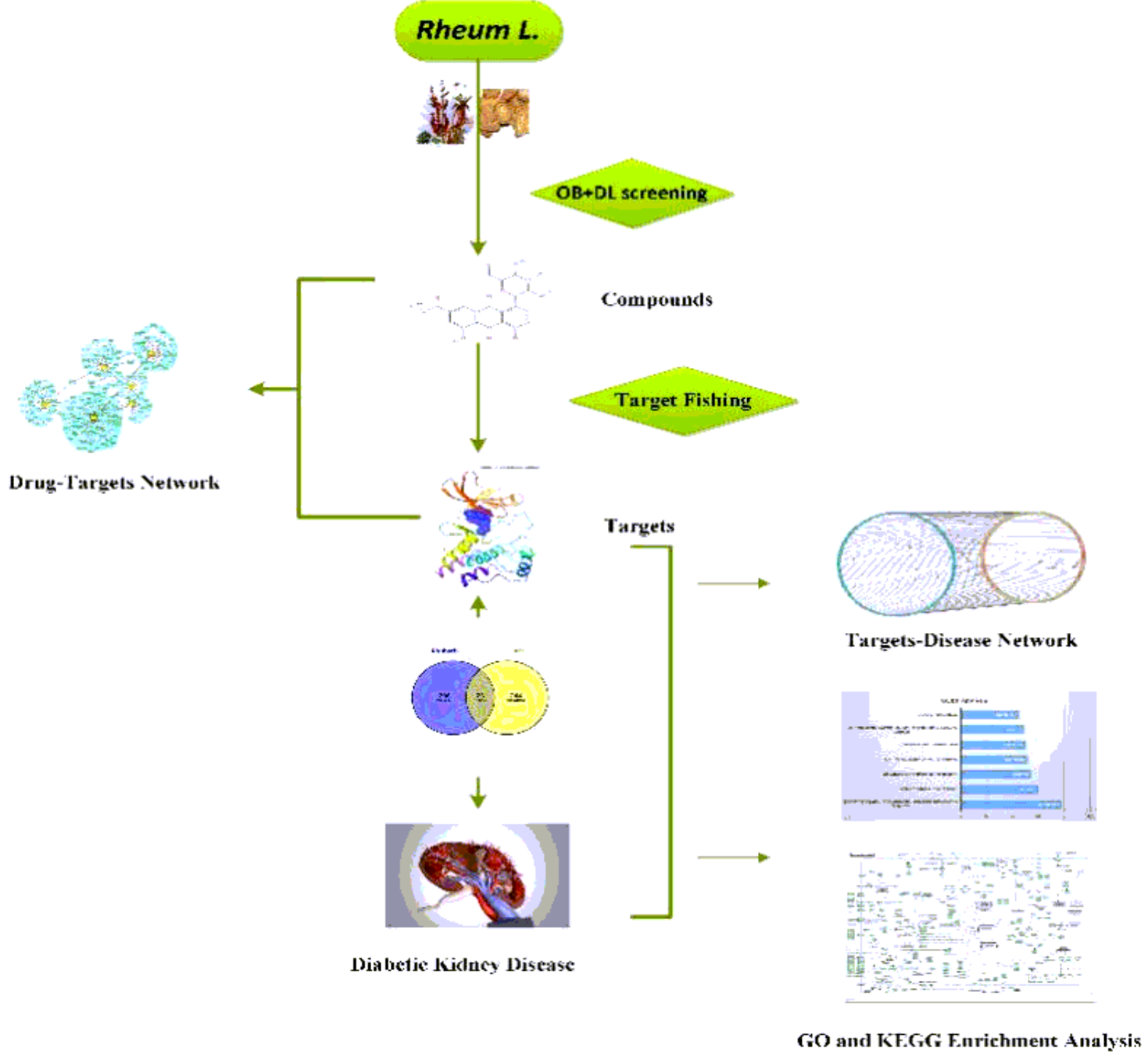

\section{Figure 1}

The flowchart of the experimental procedures 


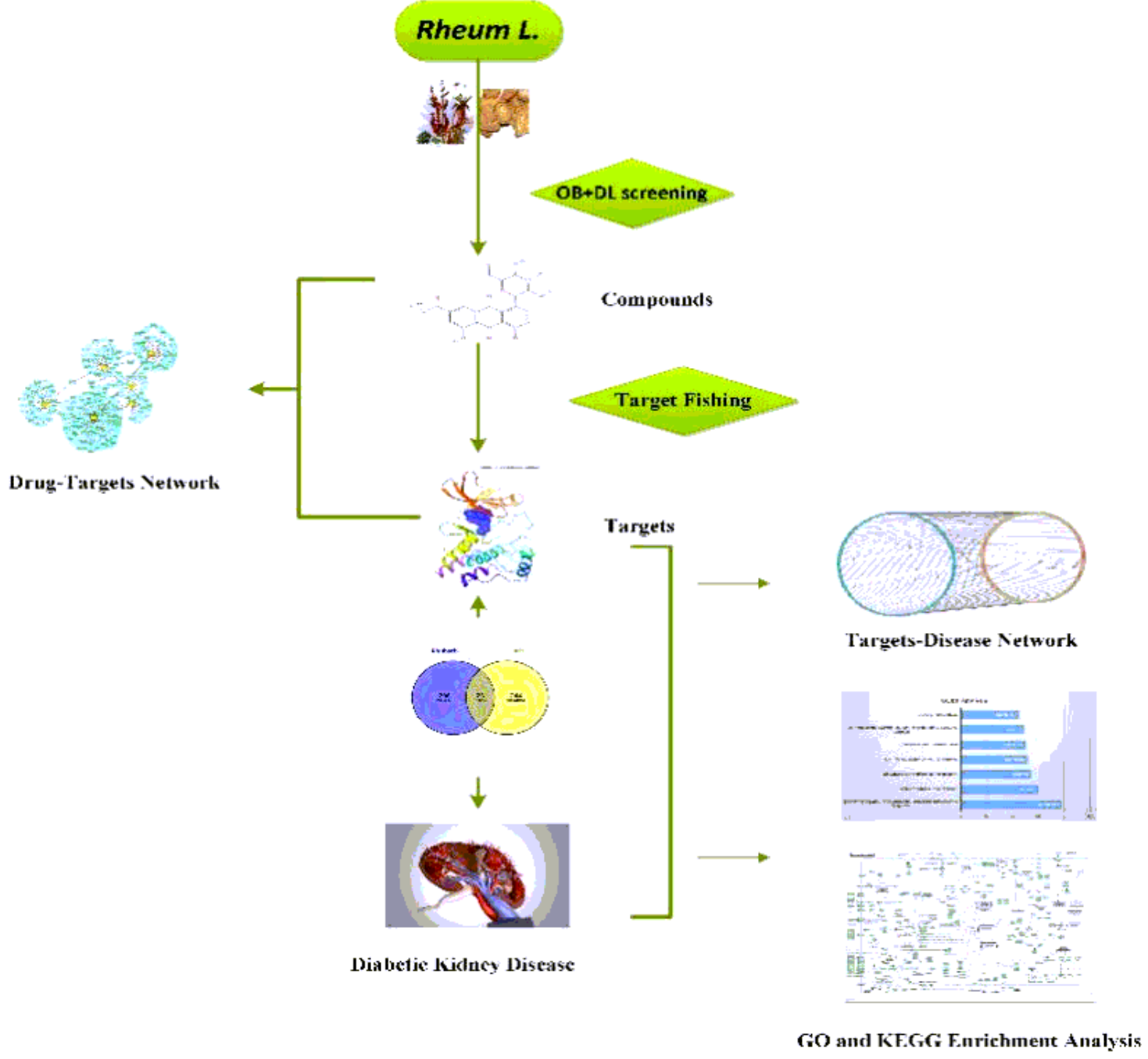

\section{Figure 1}

The flowchart of the experimental procedures 


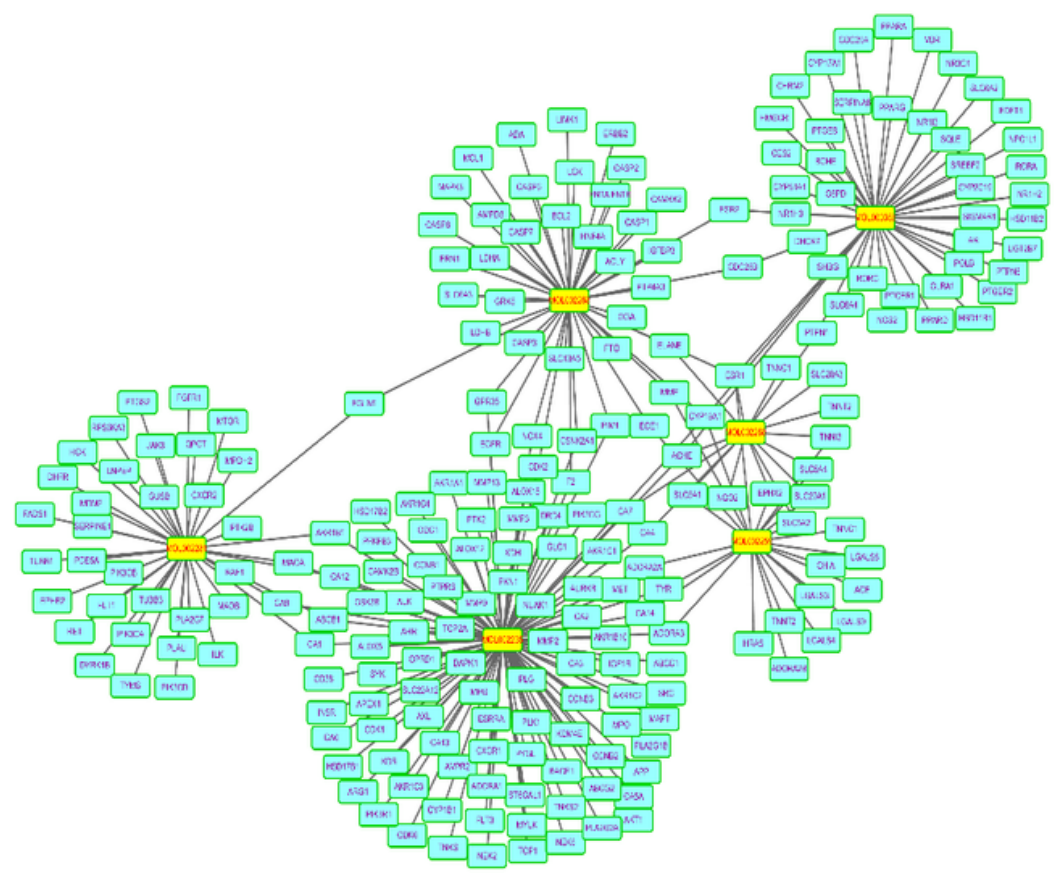

Figure 2

The C-T network that consists of 6 nodes and 271 targets. Yellow and blue nodes denote the compounds and targets, respectively.

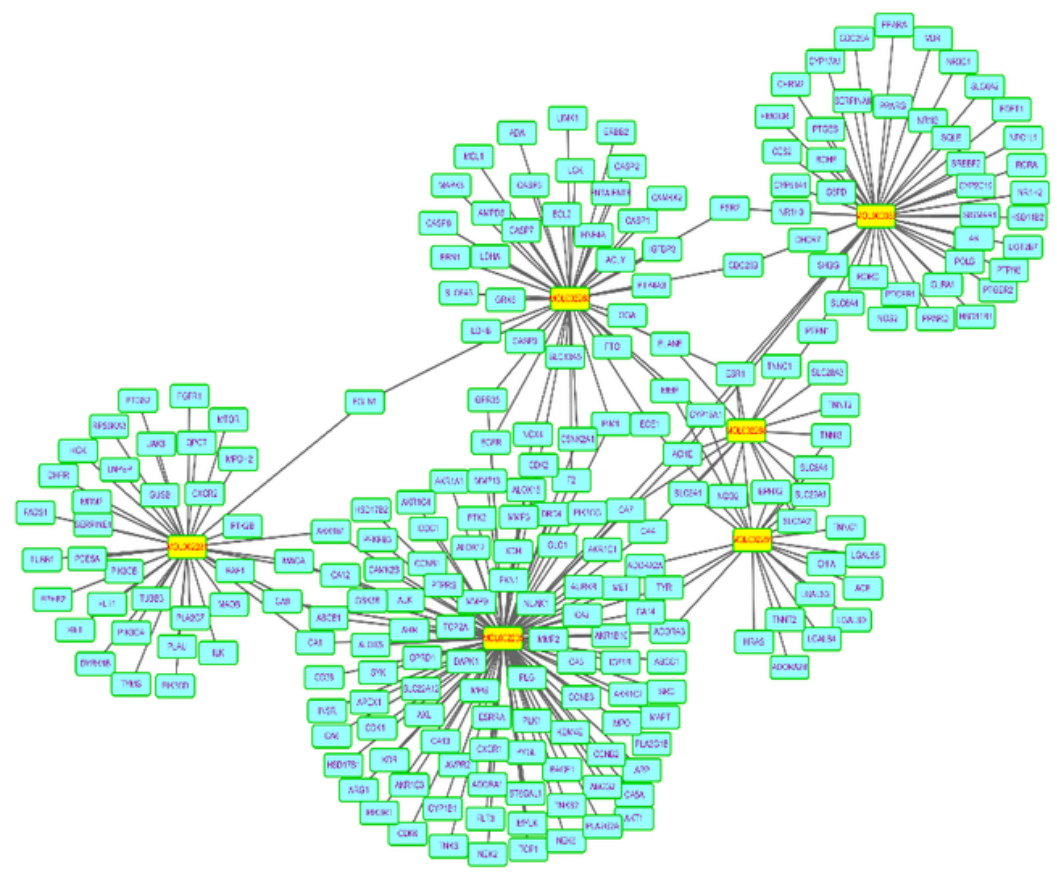

Figure 2

The C-T network that consists of 6 nodes and 271 targets. Yellow and blue nodes denote the compounds and targets, respectively. 


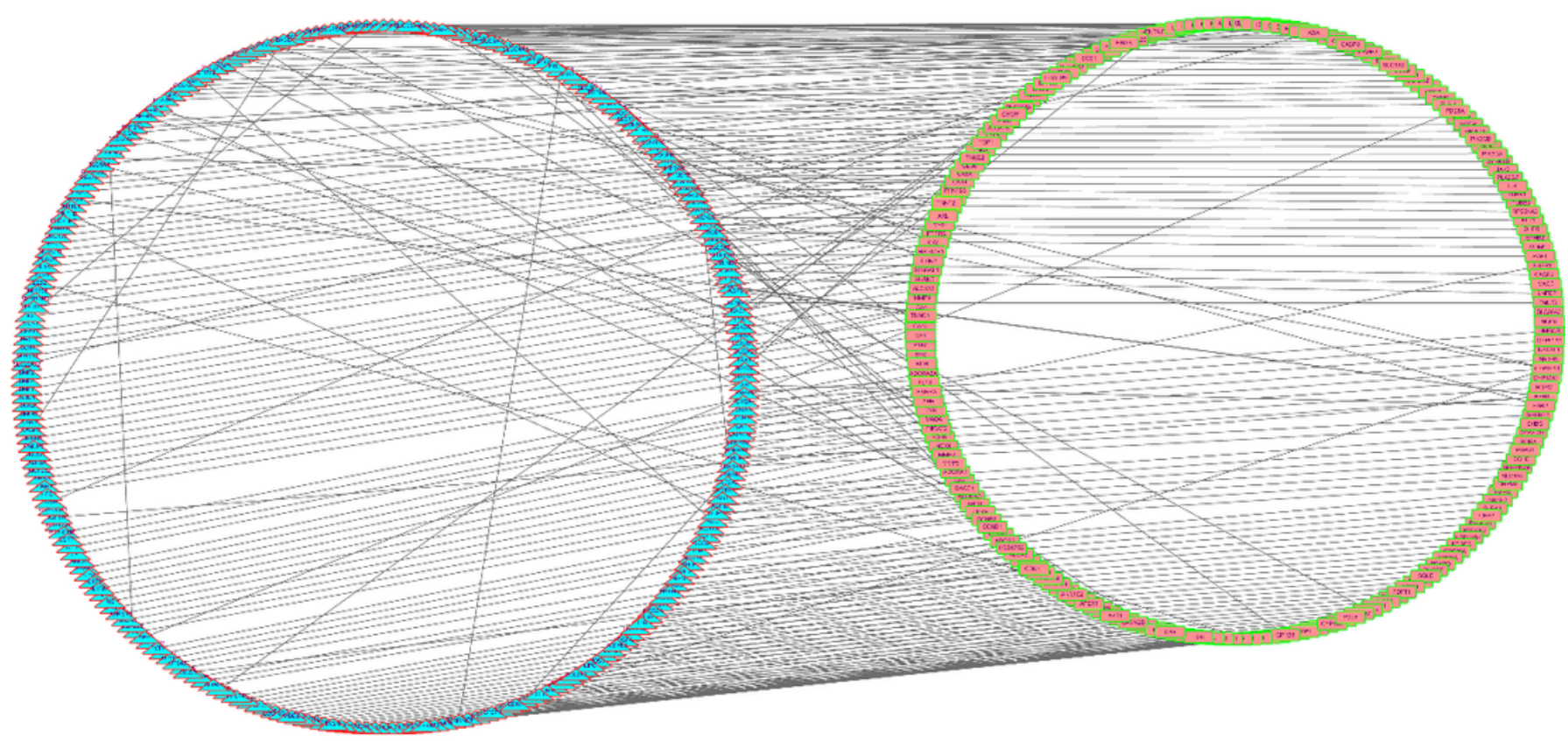

Figure 3

The T-D network that consists of 267 nodes and 271 targets. Pink and blue nodes denote the diseases and targets, respectively.

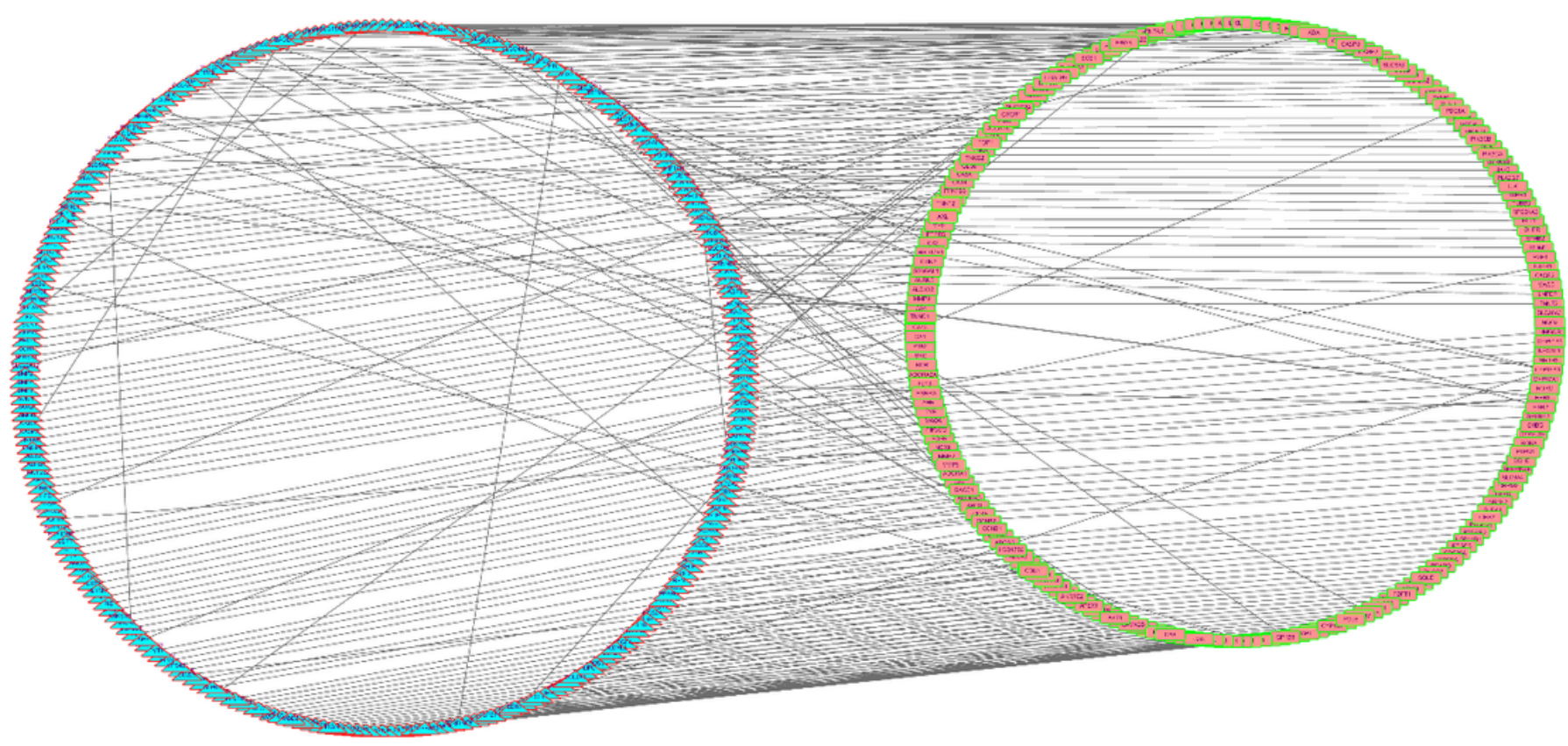

Figure 3

The T-D network that consists of 267 nodes and 271 targets. Pink and blue nodes denote the diseases and targets, respectively. 


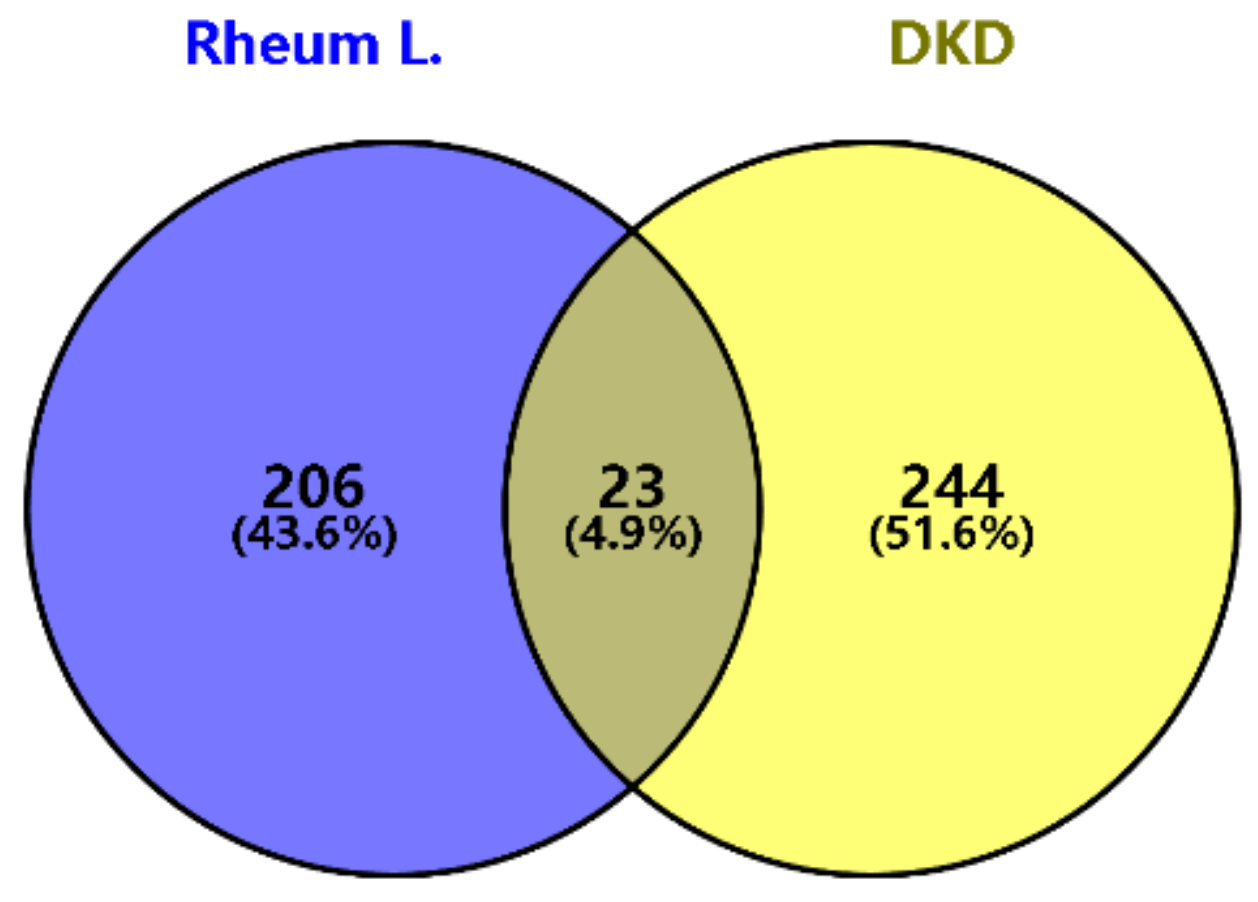

Figure 4

The 23 matching targets of the related targets in Rheum L. on DKD. 


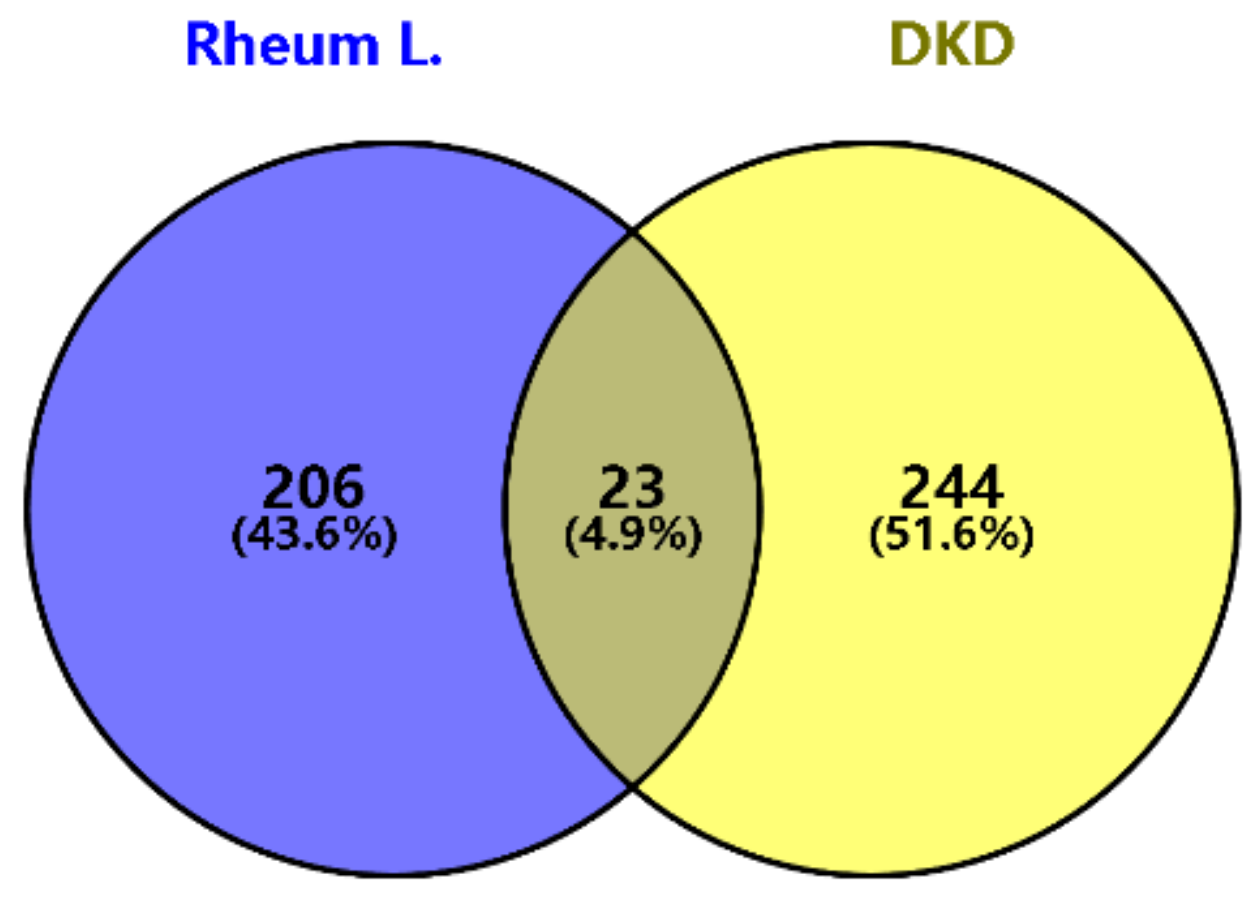

Figure 4

The 23 matching targets of the related targets in Rheum L. on DKD. 


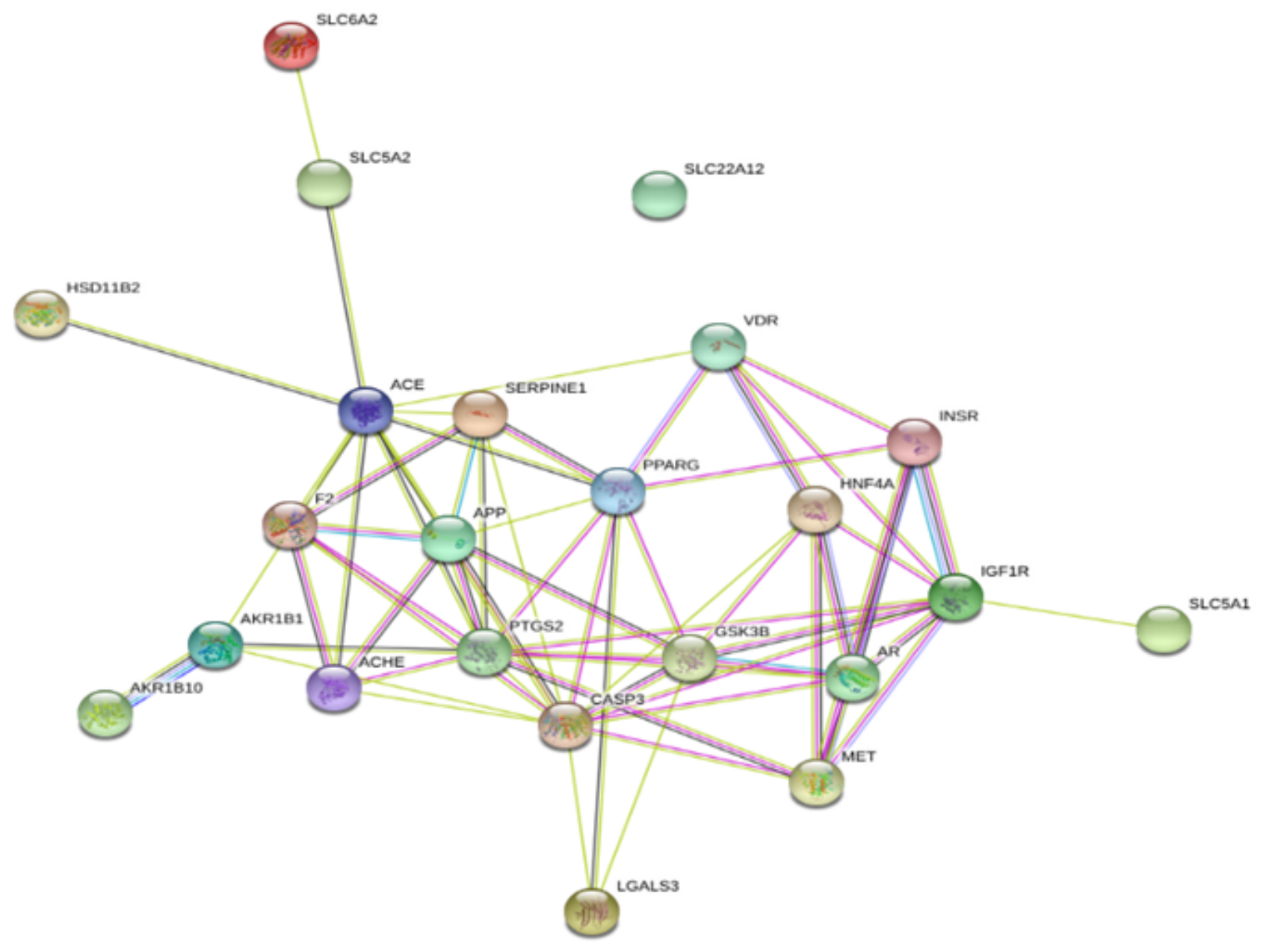

Figure 5

PPI network of 23 nodes and 65 edges established in the String database. 


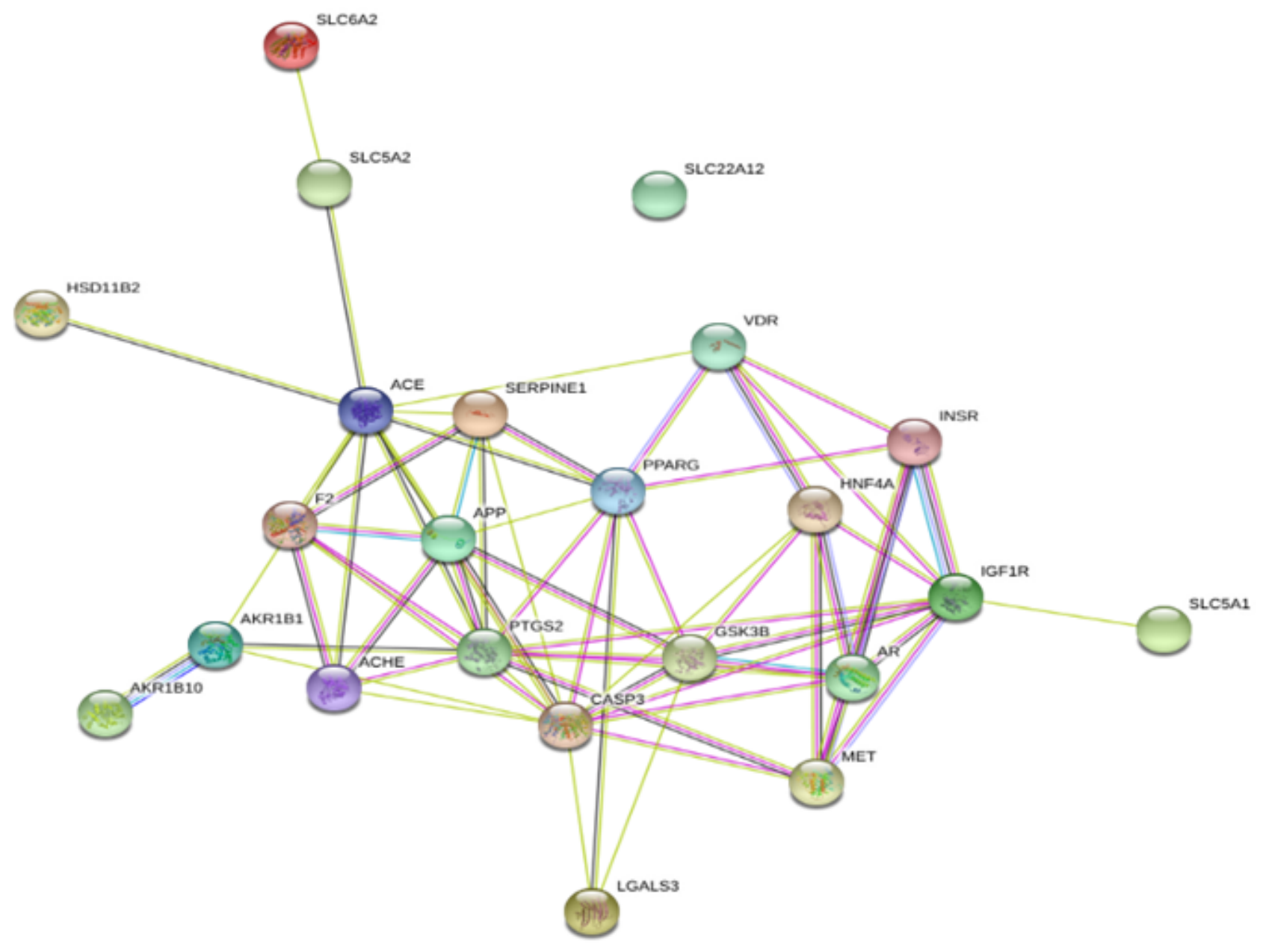

Figure 5

PPI network of 23 nodes and 65 edges established in the String database. 


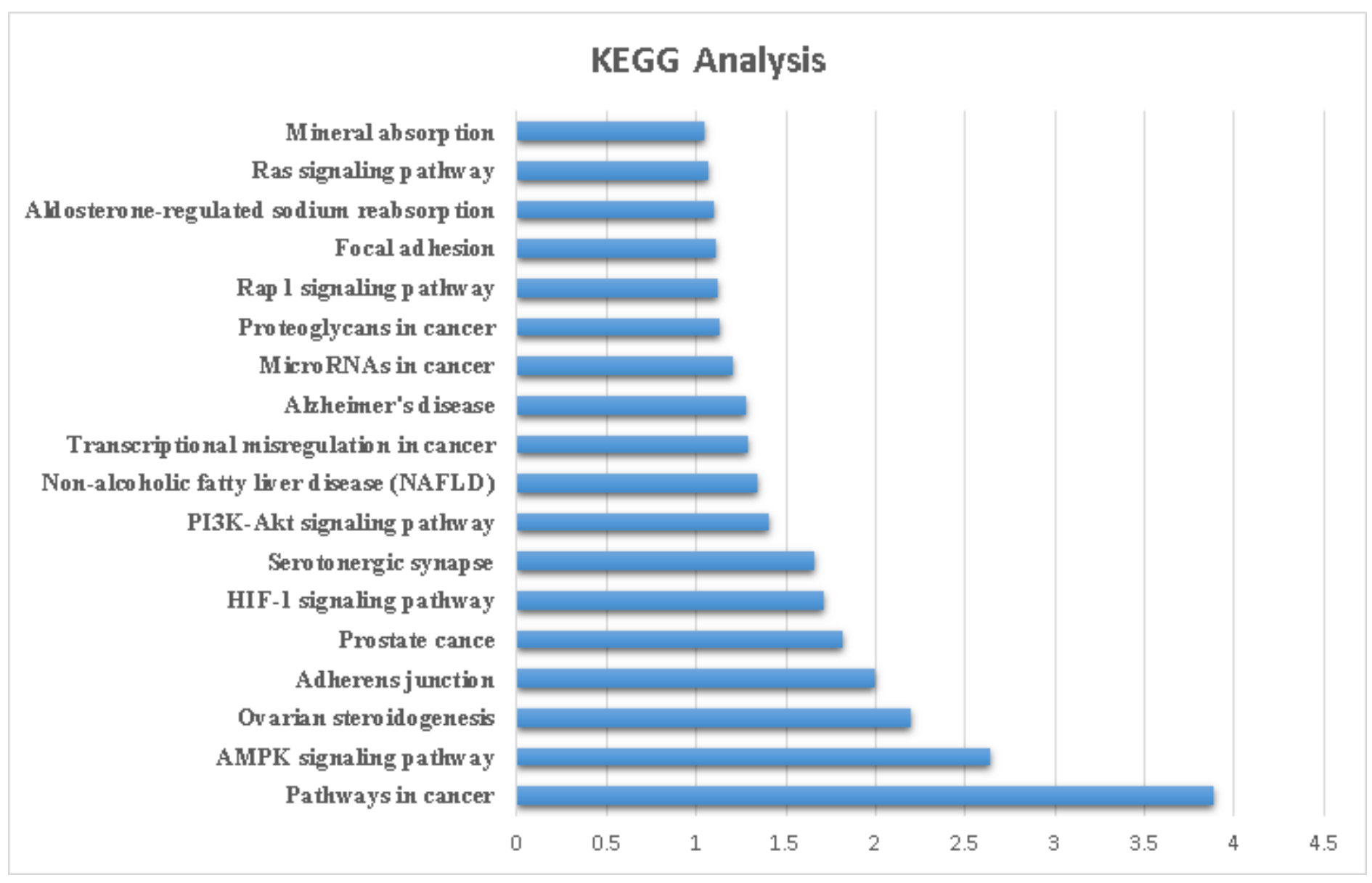

Figure 6

The main 18 pathways enriched by major hubs from the DAVID database. 


\section{KEGG Analysis}

\section{Mineral absorp tion \\ Ras signaling p atkw ay}

Alosterone-regulated sodium reabsorp tion

Focal ad hesion

Rap 1 signaling $p$ athw ay

Proteoglycans in cancer

MicroRNAs in cancer

Ahheiner's disease

Transcrip tio nal misregulation in cancer Non-alcoholic fatty lis er disease (NAFLD)

PI3K-Akt signaling $p$ atkw ay

Sero to nergic synapse HIF-1 signaling pathway

Prostate cance Adherens junction

Ovarian steroidogenesis AMPK signaling pathw ay

Pathways in cancer

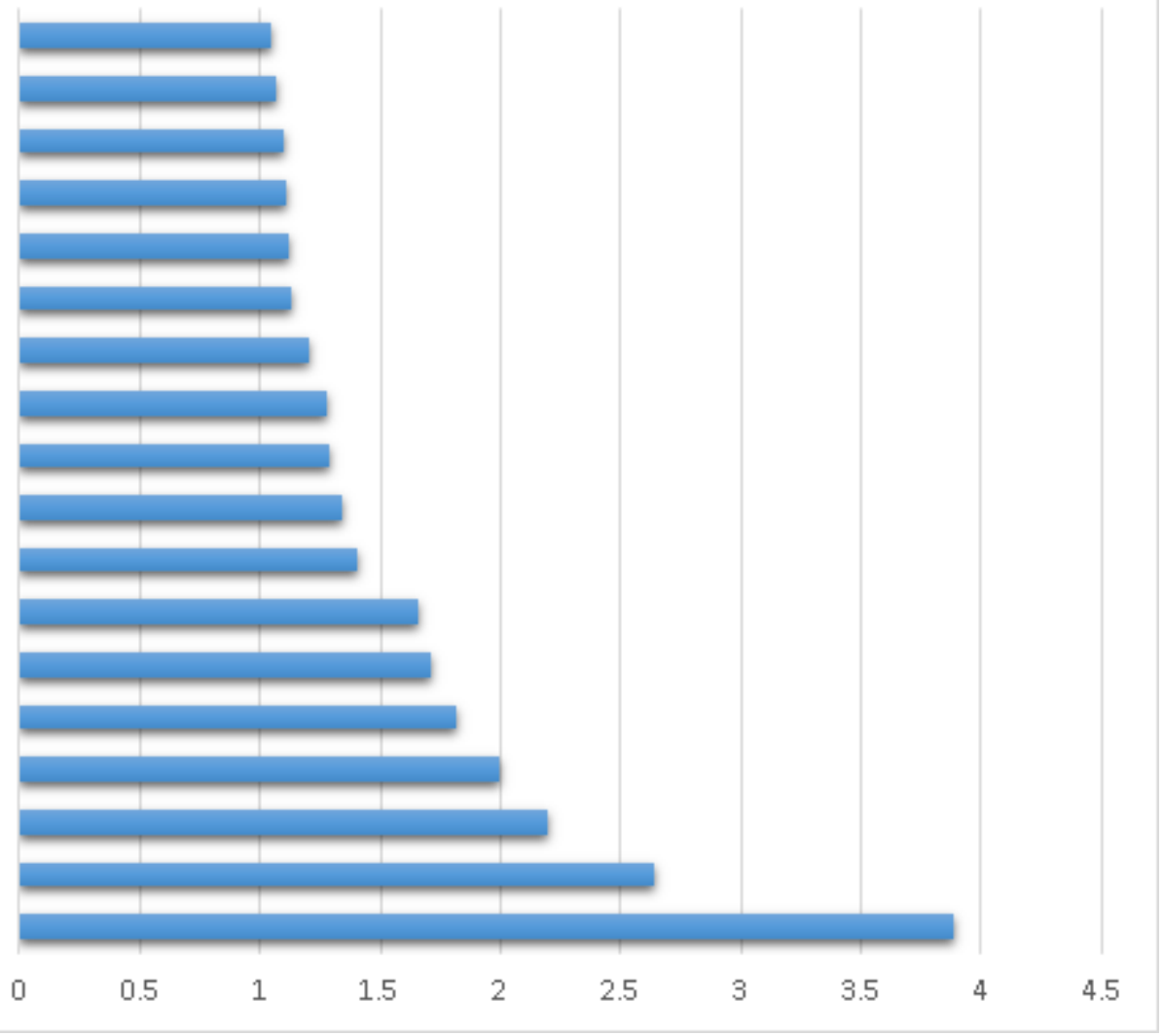

\section{Figure 6}

The main 18 pathways enriched by major hubs from the DAVID database.

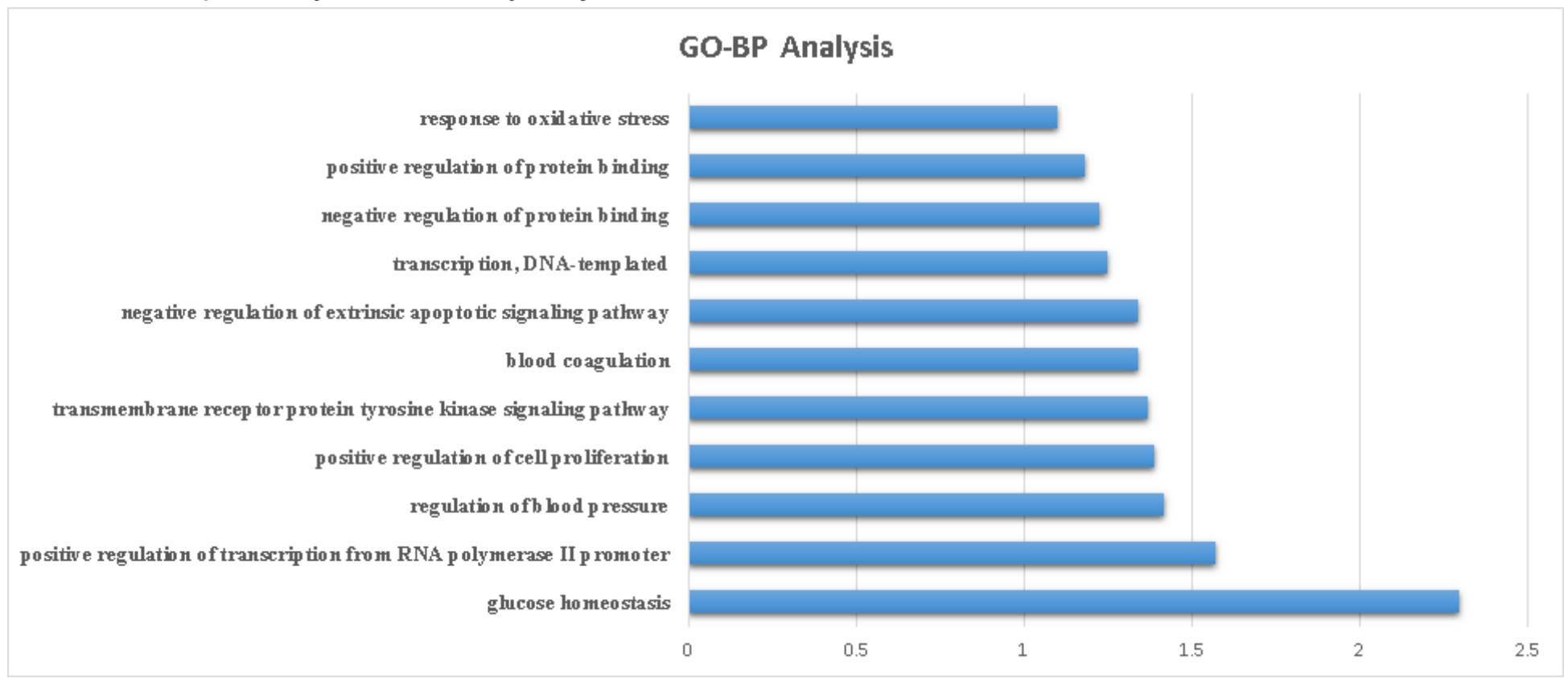

\section{Figure 7}

Main $11 \mathrm{GO}$ biological process by major hubs from the DAVID database 
GO-BP Analysis

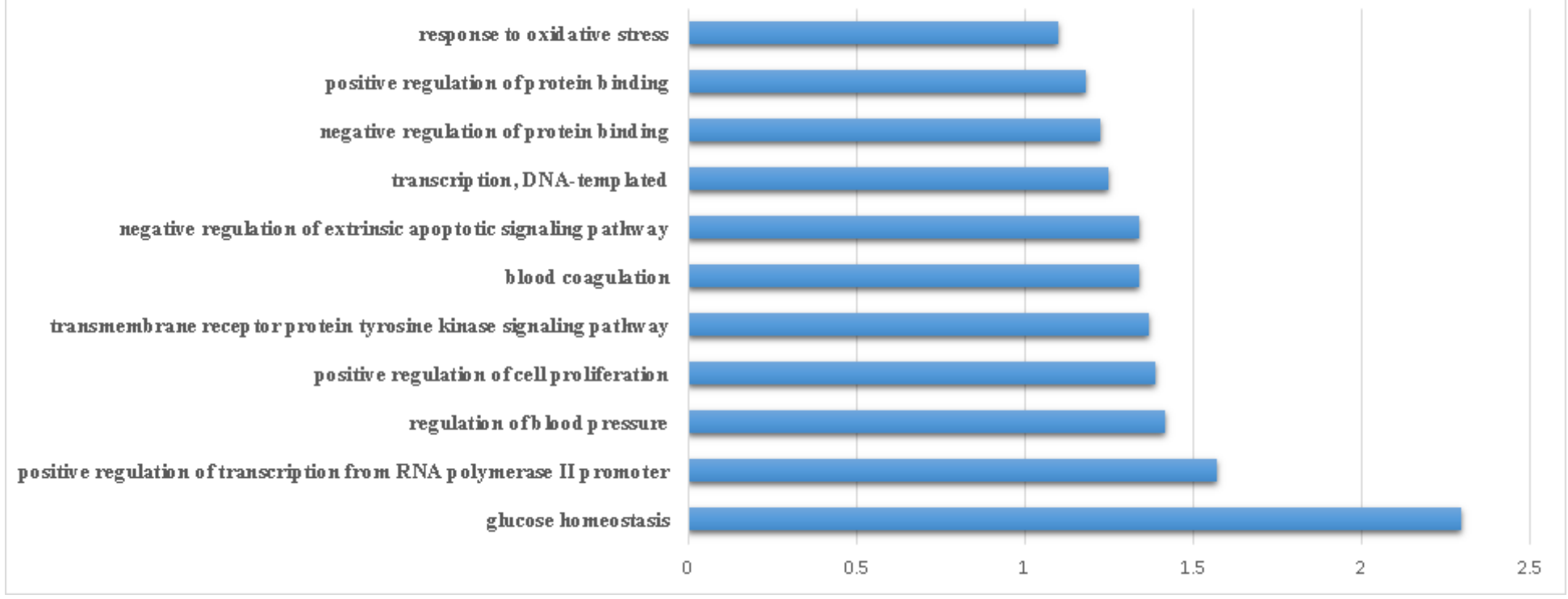

\section{Figure 7}

Main $11 \mathrm{GO}$ biological process by major hubs from the DAVID database 